\title{
Factores que determinan el estado nutricional de los estudiantes de la licenciatura de enfermería de la UAEH
}

Factors that determine the nutritional status of nursing students at UAEH María Luisa Sánchez-Padilla ${ }^{a}$, Viridiana Ramírez-Gutiérrez ${ }^{b}, J_{\text {osé Arias-Rico }}{ }^{\text {, }}$ Reyna Cristina Jiménez-Sánchez ${ }^{d}$, Margarita Lazcano-Ortiz ${ }^{e}$, Karina Chávez-Rubio ${ }^{f}$, Alma Belem López-Escudero ${ }^{g}$ y Jossajandy Pérez-Hernández ${ }^{h}$

\begin{abstract}
:
Objective: To identify the factors that lead to a deterioration in the nutritional status of the students of the Nursing Degree. Material and Method: A non-experimental, cross-sectional study was conducted, the sample was 30 students of the nursing degree. Results: Despite the factors that contribute to poor diet due to the short time that our population has, only $10 \%$ are obese, while $33.3 \%$ tend to be overweight with a small difference in normal status, which we indicates that most of them are appropriate to their weight and height that corresponds to them with a percentage of $56.7 \%$. Conclusion: When carrying out our research we can conclude that the students of 7th and 8th of the Nursing Degree of the UAEH, our population showed normal levels in their nutritional status which indicates that at the beginning of the Degree their weight is within of the parameters established according to the Secretary of Health and during their academic training students show a minimum variation of initial weight to the current one, resulting in $60 \%$ of the students surveyed have a body mass index within the appropriate limits to Your weight, size and age. The nutritional level of the surveyed students makes us mention that $60 \%$ have a regular diet because the students consume their food inside and outside the Institute which lead them to nutritional deterioration due to the low intake of nutrients and food healthy adding the lack of recurring physical activity due to excessive work and lack of time.

Keywords:

Nutritional status, Bachelor's students, Body mass index.

Resumen:

Objetivo: Identificar los factores que conllevan a un deterioro en el estado nutricional de los estudiantes de la Licenciatura en enfermería. Material y Método: Se realizo un estudio no experimental, transversal, la muestra fueron 30 alumnos de la licenciatura en enfermería. Resultados: Se considera que a pesar de los factores que contribuyen a una mala alimentación por el escaso tiempo que tiene nuestra población solo un $10 \%$ presenta obesidad, mientras tanto un $33.3 \%$ tiende a tener sobrepeso con una diferencia escasa del estatus normal, que nos indica que la mayoría de ellos se encuentras adecuados a su peso y talla que les corresponde con porcentaje de 56.7\%. Conclusión: Al realizar nuestra investigación podemos concluir que los alumnos de $7^{\circ} \mathrm{Y} 8^{\circ}$ de la Licenciatura en enfermería de la $\mathrm{UAEH}$, nuestra población arrojo niveles normales en su estado nutricional el cual nos indica que al inicio de la Licenciatura su peso se encuentra dentro de los parámetros establecidos de acuerdo a la Secretaria de Salud y durante su formación académica los alumnos muestran una variación mínima de peso inicial al actual dando como resultado que el $60 \%$ de los alumnos encuestados presentan un índice de masa corporal dentro de los límites adecuados a su peso, talla y edad. El nivel nutricional que cursan los alumnos encuestados nos hace mención que el $60 \%$ tiene una alimentación regular debido a que los alumnos consumen sus alimentos dentro y fuera del Instituto lo cual los conllevan a un deterioro nutricional a causa a la poca ingesta de nutrientes y alimentos sanos sumándole la falta de actividad física recurrente debido al exceso de trabajo y la falta de tiempo.
\end{abstract}

Palabras Clave: Estado nutricional, Estudiantes de la licenciatura, Índice de masa corporal.

\footnotetext{
Autor de Correspondencia, Universidad Autónoma del Estado de Hidalgo, Instituto de Ciencias de la Salud,

https://orcid.org/0000-0001-7621-0993,Email: marialuisasanchezpadilla@yahoo.com

${ }^{\mathrm{b}}$ Universidad Autónoma del Estado de Hidalgo, Instituto de Ciencias de la Salud, https://orcid.org/0000-0002-2133-0063,

Email: viri_gutierrez16@hotmail.com

Universidad Autónoma del Estado de Hidalgo, Instituto de Ciencias de la Salud, http://orcid.org/0000-0003-0219-0410, Email: josearias.rico@hotmail.com

${ }^{\mathrm{d}}$ Universidad Autónoma del Estado de Hidalgo, Instituto de Ciencias de la Salud, https://orcid.org/0000-0001-9264-8514, Email: cristyji@hotmail.com

e Universidad Autónoma del Estado de Hidalgo, Instituto de Ciencias de la Salud, https://orcid.org/0000-0002-9653-9291, Email: magos1000@hotmail.com

${ }^{\mathrm{f}}$ Universidad Autónoma del Estado de Hidalgo, Instituto de Ciencias de la Salud, http://orcid.org/0000-0001-5719-1854, Email: kary070895@hotmail.com

g Universidad Autónoma del Estado de Hidalgo, Instituto de Ciencias de la Salud, https://orcid.org/0000-0003-4578-6441, Email: belem_0924@hotmail.com

h Universidad Autónoma del Estado de Hidalgo, Instituto de Ciencias de la Salud, https://orcid.org/0000-0002-4767-9431,

Email: jossajandyperez@gmail.com
} 


\section{Introducción}

En la presente investigación observaremos el estilo de vida alimenticio de los alumnos el séptimo semestre grupo de la Licenciatura en Enfermería del Instituto de Ciencias de la Salud de la Universidad Autónoma del Estado de Hidalgo ubicado en Circuito Ex Hacienda La Concepción S/N Carretera Pachuca-Actopan, San Agustín Tlaxiaca Hidalgo. Estado nutricional es la situación en la que se encuentra una persona en relación con la ingesta y adaptaciones fisiológicas que tienen lugar tras el ingreso de nutrientes. Evaluación del estado nutricional será por tanto la acción y efecto de estimar, apreciar y calcular la condición en la que se halle un individuo según las modificaciones nutricionales que se hayan podido afectar. La evaluación nutricional mide indicadores de la ingesta y de la salud de un individuo o grupo de individuos, relacionados con la nutrición. Pretende identificar la presencia, naturaleza y extensión de situaciones nutricionales alteradas, las cuales pueden oscilar desde la deficiencia al exceso. El estado nutricional no solamente está determinado por talla y peso, sino además por indicadores clínicos y bioquímicos. La adultez represente el largo periodo que se sitúa entre crecimiento y desarrollo activos de las fases de lactancia infancia y adolescencia las bases del adulto mayor donde una de las preocupaciones es sostener la capacidad física y mental.

La adultez temprana, por lo general, alcanzar los 20 años de edad implica volverse independientes, dejar el hogar paterno, terminar la educación, formal iniciar un trabajo regular y empezar el desarrollo profesional, forma relaciones y elegir a una pareja, para muchas personas planear, comprar y preparar las comidas son habilidades que apenas se empiezan a desarrollar. Alcanzar los 30 años puede caracterizarse por la adquisición de mayores responsabilidades, incluyendo tener hijos, proveer y cuidar una familia, consolidar una carrera y participar en asuntos comunitarios y cívicos.

Diversos autores han destacado que la población universitaria es un grupo especialmente vulnerable desde el punto de vista nutricional, ya que se caracteriza por: saltarse comidas con frecuencia, picar entre horas, tener preferencia por comida rápida y consumir alcohol frecuentemente. El periodo de estudios universitarios suele ser el momento en que los estudiantes asumen por primera vez la responsabilidad de su comida. Por tanto, se trata de un periodo de educación crítico para el desarrollo de hábitos dietéticos que tienen mucha importancia en la futura salud. (M. Arroyo Izaga ${ }^{*}$, 2014) Teniendo en cuenta además que más de un millón de estudiantes están matriculados, este colectivo constituye un grupo de población lo suficientemente numeroso e interesante como para tratar de reducir la prevalencia de sobrepeso en la vida adulta a través de estrategias de promoción de la salud. (M. Arroyo lzaga*, 2014)

En los últimos años los estudios epidemiológicos sobre la dieta y la salud, incluyendo la obesidad, han cambiado su orientación. Antes se enfocaban hacia un único nutriente, por ejemplo, la grasa dietética, mientras que ahora lo hacen hacia la calidad de la dieta total y el patrón dietético. (M. Arroyo Izaga*, 2014)

Para el estudio de la calidad de la dieta y la salud se han diseñado diversos índices, como, por ejemplo: el índice de calidad de la dieta, el índice de diversidad de la dieta, y el índice de alimentación saludable. Algunos autores han estudiado los patrones de ingesta dietética, y han descrito los efectos de la calidad de la dieta sobre la salud. Pero, teniendo en cuenta estos estudios, la etiología nutricional de la obesidad continúa siendo controvertida. (M. Arroyo Izaga*, 2014)

El sobrepeso y la obesidad son factores de riesgo para enfermedades como: asma, cáncer y enfermedades coronarias. Se estima que la prevalencia de obesidad en la población general de algunos países, es del 30\%; sin embargo, esta cifra varía de acuerdo al país, al grupo de edad y al sexo. En jóvenes universitarios se ha reportado que el índice de masa corporal (IMC) en hombres tiene una media entre 22 y $24 \mathrm{~kg} / \mathrm{m} 2$ y en las mujeres varía entre 22 a 26.5 $\mathrm{kg} / \mathrm{m} 2$; la prevalencia de obesidad se presenta en poco menos de $5 \%$. Uno de los indicadores más usados en la detección del sobrepeso y la obesidad es el IMC. Éste se obtiene al dividir el peso en kilogramos sobre la estatura del individuo en metros cuadrados; con este criterio se acepta que las personas con sobrepeso son aquellas que se encuentran entre 25 a $29.99 \mathrm{~kg} / \mathrm{m} 2$ y las obesas se consideran cuando este índice es igual o mayor de $30 \mathrm{~kg} / \mathrm{m} 2$. Otro indicador de bajo costo y fácil aplicación es la percepción que la persona tiene de su imagen corporal. Este procedimiento ha mostrado una correlación por arriba de 0.6 con respecto al IMC, por lo que es un instrumento adecuado a estudios de campo. El estudio de la imagen corporal, conceptualizada por el propio individuo, se encuentra ligada a la identidad, sentimiento y conductas del mismo. (Leoncio Miguel Rodríguez Guzmán)

En México la salud es un derecho, por lo que los estados de mala nutrición desnutrición, sobrepeso u obesidad deben ser prevenidos o diagnosticados y tratados sin importar la edad de quienes los padecen, debido a que constituyen un factor de riesgo para otras patologías. Sin embargo, se debe reconocer la importancia de invertir especialmente en los grupos que constituirán en los próximos años a la población productiva y económicamente activa, debido a que serán un pilar fundamental para México. Así mismo, se sabe que en la actualidad predominan las enfermedades no transmisibles como el sobrepeso y la obesidad (prevalencia del $70 \%$ en 
adultos), por encima de la desnutrición (prevalencia menor a $2 \%$ en adultos), que en la década de los cincuentas encabezaba las listas de la morbi-mortalidad nacional, con una prevalencia del $61.8 \%$. Aunado a ello, se ha evidenciado que los patrones socioculturales inmersos en la transición epidemiológica han modificado significativamente la imagen corporal, entendiendo la delgadez, incluso extrema, como un patrón normal, por lo que se ha incrementado la prevalencia de trastornos de la conducta alimentaria, especialmente en adolescentes y adultos jóvenes, que ocasionan procesos de desnutrición de energía, proteína y diversos nutrimentos asociada a estos trastornos. Por otro lado, existen evidencias de que los hábitos de alimentación son factores modificables que intervienen en la expresión o el desarrollo de diversas patologías tanto por deficiencia como por exceso de energía y nutrimentos. Por ello diversas enfermedades crónicodegenerativas no transmisibles pueden prevenirse si se lleva a cabo un estilo de vida saludable. Todo esto es indispensable, ya que, al conocer las modificaciones de la prevalencia nacional de estas patologías, es posible pronosticar el daño, establecer estrategias o acciones que disminuyan su prevalencia y evaluar el efecto de dichas acciones. Debido a que se ha demostrado que la prevención es la mejor alternativa para disminuir la mortalidad de estas enfermedades a lo largo de su historia natural y a que constituyen uno de los mayores retos que enfrenta el sistema de salud, la política nacional está actuando sobre los determinantes de la salud mediante el fortalecimiento de la promoción de la salud, la prevención de enfermedades y control de riesgos sanitarios, con el objeto de reducir el impacto que tienen las enfermedades y las lesiones sobre los individuos, familias, comunidades y la sociedad en su conjunto. Dentro del marco de la promoción de la salud, la Escuela de Dietética y Nutrición de ISSSTE se ha incorporado al desarrollo del modelo de las Escuelas Promotoras de la Salud impulsado por la Organización Panamericana de la Salud (OPS), la Organización Mundial de la Salud (OMS) y el Programa Sectorial de Salud de nuestro país, en la que se originan estrategias preventivas para favorecer la cultura de la salud y el desarrollo de oportunidades para elegir estilos de vida saludables, los cuales son considerados como patrones de comportamiento, valores y forma de vida que caracterizan a un individuo o grupo y representan las prácticas diarias, hábitos y actividades que pueden afectar la salud del individuo. Cuando estas acciones son responsables, adecuadas y positivas contribuyen a prevenir enfermedades y accidentes; también propician diferentes tipos de bienestar y buscan alcanzar estilos de vida de alta calidad. Algunos de los comportamientos que forman parte del estilo de vida saludable son: llevar una alimentación y nutrición adecuada, realizar actividad física y actividades recreativas de forma regular, controlar el estrés, no fumar o abusar de las bebidas alcohólicas, cafeína o fármacos y adoptar medidas de higiene y seguridad adecuadas. Una forma de promover los estilos de vida saludables es mediante la instrumentación de políticas alimentarias y nutricionales, así como de la educación y orientación alimentaria, las cuales son fundamentales para afrontar los problemas de salud pública en materia de alimentación y nutrición, que involucra una amplia gama de estados clínicos que oscilan desde los estados marginales leves, moderados o graves- hasta los estados de exceso de energía, nutrimentos o ambos. Sin embargo, para llevar a cabo estas actividades es indispensable obtener, en primera instancia, un diagnóstico nutricional de la población en la que se pretende actuar con el fin de identificar la mala nutrición. Así mismo, se deben conocer los hábitos de alimentación y los diversos componentes del estilo de vida para establecer las estrategias adecuadas encaminadas a promover un estilo de vida saludable y reducir el riesgo de desarrollar enfermedades crónicas. Por ello, la Escuela de Dietética y Nutrición busca detectar en sus estudiantes estados de mala nutrición en etapas tempranas, tanto por deficiencia como por exceso, para evitar, mediante una intervención nutricia, el desarrollo y progresión de enfermedades crónicas utilizando diversas técnicas de la evaluación del estado de nutrición. El objetivo de este trabajo fue identificar la presencia de mala nutrición, desnutrición, sobrepeso y obesidad en alumnos de la generación 2008 de licenciatura en dietética y nutrición mediante la evaluación del estado de nutrición, con el fin de promover un estilo de vida saludable. (Irazú Gallardo Wong, 2012)

\section{Objetivo}

Identificar los factores que conllevan a un deterioro en el estado nutricional de los estudiantes de la Licenciatura en enfermería.

\section{Material y Métodos}

Se utilizó como instrumento una encuesta donde se valora peso, talla y el tipo de alimentación que realizan, el instrumento cuenta con 16 ítems. La muestra fue de 30 alumnos de la licenciatura en enfermería, los cuales fueron 20 alumnos de séptimo y 10 de octavo semestre. El tipo de investigación es de tipo cualitativa de diseño no experimental.

\section{Resultados}

Finalmente se considera que la mayor prevalencia de edad en nuestra población es de 21 años, teniendo una frecuencia 11 y un porcentaje de $36.7 \%$, así con el peso al inicio de la carrera se mantiene en un rango de $64-68 \mathrm{~kg}$, teniendo una frecuencia 5 y su porcentaje de 25\%, comprándolo con el peso actual existen una ligera modificación en su rango con más-menos $3 \mathrm{~kg}$ de diferencia teniendo una frecuencia y 
porcentaje casi similares. En la talla pues se mantiene igual que cuando comenzaron la carrera, ya que la etapa de vida que se encuentran no suele haber cambios, manteniendo una prevalencia en la estatura de $1.55-1.60 \mathrm{~cm}$, con una frecuencia y porcentaje iguales. Arrojándonos que con estos datos la mayoría de los alumnos con un $56.7 \%$ se encuentran en su peso adecuado según su talla, teniendo un IMC normal.

Tabla 1: Índice de masa corporal en estudiantes de enfermería

\begin{tabular}{|ll|l|l|l|l|}
\hline Variable & Frecuencia & Porcentaje & Media & DE$^{*}$ \\
\hline Normal 18.5-24.9 & 17 & 56.7 & 1.53 & .681 \\
Sobrepeso 25- & 10 & 33.3 & & \\
29.9 & 3 & 10.0 & & \\
Obesidad 1 30- & & & & \\
34.9 & & & & & \\
\hline
\end{tabular}

Se considera que a pesar de los factores que contribuyen a una mala alimentación por el escaso tiempo que tiene nuestra población solo un $10 \%$ presenta obesidad, mientras tanto un $33.3 \%$ tiende a tener sobrepeso con una diferencia escasa del estatus normal, que nos indica que la mayoría de ellos se encuentras adecuados a su peso y talla que les corresponde con porcentaje de $56.7 \%$.

Tabla 2: Donde consumen sus alimentos

\begin{tabular}{|l|l|l|l|l|}
\hline VARIABLE & FRECUENCIA & PORCENTAJE & MEDIA & DE* $^{*}$ \\
\hline Instituto & 14 & 46.7 & 1.63 & .669 \\
Fuera del & 13 & 43.7 & & \\
instituto & 3 & 10.0 & & \\
Otro lugar & & & & \\
\hline
\end{tabular}

Finalmente se atribuye que por el tiempo que llevan dentro la institución la mayoría consume sus alimentos dentro de la institución con un porcentaje de $46.7 \%$, pero con una mínima de diferencia algunos la llevan fuera de la institución, ya que a los alrededores hay puestos de comida y suele ser más económico consumir fuera, mientras que solo un $10 \%$ lo realiza en otros lugares.

Tabla 3: Número de ingestas en la estadía en el instituto"

\begin{tabular}{|l|l|l|l|l|}
\hline VARIABLE & FRECUENCIA & PORCANTAJE & MEDIA & DE $^{*}$ \\
\hline 1 & 22 & 73.3 & 1.30 & .535 \\
2 & 7 & 23.3 & & \\
Ninguno & 1 & 3.3 & & \\
\hline
\end{tabular}

Se considera que la mayoría de ellos solo consume una vez alimentos durante su estancia dentro de la institución, ya sea por distintas situaciones que los lleve a eso, con una prevalencia de $73.3 \%$, mientras que un $23,3 \%$ suele realizar dos ingestas y con una mínima que no realiza ninguna.
Tabla 4: Tipo de alimentación

\begin{tabular}{|l|l|l|l|l|}
\hline VARIABLE & FRECUENCIA & PORCENTAJE & MEDIA & DE $^{*}$ \\
\hline Regular & 15 & 50.0 & 1.93 & .980 \\
Buena & 2 & 6.7 & & \\
Mala & 12 & 43.3 & & \\
\hline
\end{tabular}

En la tabla nos indica como ellos consideran su alimentación que llevan, sobresaliendo la mayoría dice que su alimentación es regular con un 50\%, y que solo un $6.7 \%$ tiene una buena ingesta de alimentos, mientras que la tercera parte con una mínima de diferencia tiene una mala alimentación con un porcentaje de $35 \%$, por lo que nos damos cuenta que por eso algunos presentan sobrepeso $\mathrm{u}$ obesidad.

Tabla 5: Consumo de frutas y verduras"

\begin{tabular}{|l|l|l|l|l|}
\hline VARIABLE & FRECUENCIA & PORCANTAJE & MEDIA & DE $^{*}$ \\
\hline $1-3$ veces & 20 & 66.7 & 1.37 & .556 \\
$4-5$ veces & 9 & 30.0 & & \\
Nunca & 1 & 3.3 & & \\
& & & & \\
\hline
\end{tabular}

En la tabla nos indica el consumo de frutas y verduras, qué la mayoría nos hace referencia que consumo por lo menos de 1 a 3 veces a la semana, y teniendo una diferencia de casi más de la mitad solo consume de 4 a 5 veces y solo una mínima que no consume ninguna fruta ni verdura durante la semana con un porcentaje de $3.3 \%$.

Tabla 6: Consumo de agua"

\begin{tabular}{|l|l|l|l|l|}
\hline VARIABLE & FRECUENCIA & PORCANTAJE & MEDIA & DE $^{*}$ \\
\hline 500ml-1 litro & 1 & 3.3 & 1.97 & .183 \\
1.5 litros-2 litros & 29 & 96.7 & & \\
Más de 3 litros & 0 & 0.0 & & \\
& & & & \\
\hline
\end{tabular}

Finalmente se considera que la mayoría consume de 1.5 a 2 litros de agua por día, a lo que se llega es que un $96.7 \%$ tiene una buena hidratación y que solo un 3.3\% ingiere menos de 1 litro por día, ya sea por diferentes cuestiones que lo lleve a hacer tan poco ingesta durante el trascurso del día.

Tabla 7: Activación física"

\begin{tabular}{|l|l|l|l|l|}
\hline VARIABLE & FRECUENCIA & PORCENTAJE & MEDIA & DE* $^{\star}$ \\
\hline $1-3$ veces & 14 & 46.7 & 1.97 & .964 \\
Diario & 3 & 10.0 & & \\
Nunca & 13 & 43.3 & & \\
& & & & \\
& & & & \\
\hline
\end{tabular}

En la tabla nos indica que tan frecuente realizan actividad física, lo que nos muestra que casi la mayoría son persona 
sedentaria a que no llevan ningún tipo de ejercicio durante la semana, con una prevalencia de $43.3 \%$, mientras que con casi más de la mitad por lo menos realiza de 1 a 3 veces a la semana y solo un $10 \%$ de ellos la lleva acabo diariamente.

\section{Discusión}

De acuerdo al estudio realizado por la universidad de Granada, España, en la evaluación nutricional de una población universitaria menciona que La alimentación es uno de los factores que más condiciona la salud de los individuos, ejerciendo un papel primordial sobre el desarrollo físico y el crecimiento, la reproducción y el rendimiento físico e intelectual

Por lo que relacionándolo con la tabla número 2 se muestra el índice de masa corporal, obtenido de 20 estudiantes de enfermería, que nos indica que el $60 \%$ de ellos tiene un peso adecuado a su talla, mientras que un $25 \%$ presenta sobrepeso, existiendo una mínima de $15 \%$ con obesidad.

Dentro del estudio realizado en base a la nutrición de los alumnos de $7^{\circ}$ semestre, nuestros resultados arrojaron que la alimentación dentro del instituto de ciencias de la salud es regular.

En base a la investigación de la Universidad Tecnológica Nacional (UTN), Facultad Regional Córdoba de la Ciudad de Córdoba (Argentina) referente al Estado nutricional en estudiantes universitarios: su relación con el número de ingestas alimentarias diarias y el consumo de macronutrientes La dieta y la nutrición son muy importantes para promover y mantener la buena salud a lo largo de toda la vida. Está bien establecida su función como factores determinantes de enfermedades no transmisibles (ENT) que puede ser modificado, y no cesa de crecer la evidencia científica en apoyo del criterio de que el tipo de dieta tiene una gran influencia, tanto positiva como negativa, en la salud a lo largo de la vida y que la realización de 3-4 ingestas diarias parece asociarse a un mejor aporte de energía y a un control del peso más satisfactorio. Según Leidy et al., es difícil hacer conclusiones definitivas sobre la importancia relativa de la frecuencia alimentaria per se a la regulación energética y la obesidad basada en los ensayos alimentarios controlados, llevados a cabo hasta la fecha.

Nuestra tabla numero 1 nos habla sobre las variables demográficas, obtuvimos que de una muestra de 20 alumnos encuestados, la prevalencia en edad es de 21 años, con frecuencia de 9 y un porcentaje de $45 \%$, así con el peso al inicio de la carrera se mantiene en un rango de $64-68 \mathrm{~kg}$, teniendo una frecuencia 5 y su porcentaje de $25 \%$, comprándolo con el peso actual existen una ligera modificación en su rango con más-menos $3 \mathrm{~kg}$ de diferencia teniendo la misma frecuencia y porcentaje, el indicador de la talla nos muestra que se mantiene una frecuencia de 5 en $1.49-1.54 \mathrm{~cm}$, con un porcentaje de $25 \%$. Así obteniendo el resultado que el $60 \%$ de los estudiantes tiene un IMC normal. De acuerdo La Encuesta Nacional de Salud del año 2010 incluyó 812 jóvenes de 24 años, donde se observó 10,9\% de obesidad, $79,3 \%$ de sedentarismo y que sólo $13 \%$ cumplía con las recomendaciones de consumo de verduras y frutas del Ministerio de Salud, y un estudio de 449 estudiantes universitarios de Santiago mostró bajos niveles de actividad física y de consumo de verduras y frutas. La principal barrera descrita para realizar mayor actividad física fue la limitada disponibilidad de tiempo $(68 \%)$ y para un mayor consumo de verduras y frutas "flojera" para preparar ensaladas, falta de tiempo, olvido y el hecho que estos alimentos no quitan el hambre.

Relacionando con la tabla 7 que nos indica el consumo de frutas y verduras en los 20 estudiantes de enfermería encuestados, nos hace referencia que el $55 \%$ tiene un consumo de por lo menos de 1 a 3 veces a la semana, también que solo un $45 \%$ las consume más de 4 veces y la tabla número 9 se obtiene resultados que el $60 \%$ de los estudiantes de enfermería realiza de 1 a 3 veces actividad física, y solo el $10 \%$ tiene actividad constante, mientras que el $30 \%$ son personas sedentarias

\section{Conclusión}

Al realizar nuestra investigación podemos concluir que los alumnos de $7^{\circ}$ Y $8^{\circ}$ de la Licenciatura en Enfermería de la Universidad Autónoma del Estado de Hidalgo del Instituto de Ciencias de la Salud nuestra población arrojo niveles normales en su estado nutricional el cual nos indica que al inicio de la Licenciatura su peso se encuentra dentro de los parámetros establecidos de acuerdo a la Secretaria de Salud y durante su formación académica los alumnos muestran una variación mínima de peso inicial al actual dando como resultado que el $60 \%$ de los alumnos encuestados presentan un índice de masa corporal dentro de los límites adecuados a su peso, talla y edad.

El nivel nutricional que cursan los alumnos encuestados nos hace mención que el $60 \%$ tiene una alimentación regular debido a que los alumnos consumen sus alimentos dentro y fuera del Instituto lo cual los conllevan a un deterioro nutricional a causa a la poca ingesta de nutrientes y alimentos sanos sumándole la falta de actividad física recurrente debido al exceso de trabajo y la falta de tiempo.

Gracias a nuestro instrumento se pudo obtener información de primera mano para poder cumplir nuestros objetivos e hipótesis y con esto responder a nuestra pregunta de investigación y planteamiento de problema. 


\section{Bibliografía}

1. Sakraida J. Modelo de Promoción de la Salud. Nola J Pender. En: Maerriner TA, Rayle-Alligood M, editores. Modelos y teorías de enfermería, $6^{\circ}$ ed. España: Elsevier-Mosby; 2007.

2. Worchel S. Cooper J, Goethals GR. Olson J. Psicología Social. Madrid: thomson Editores 2002.

3 Bowman, B. A. (2014). Conocimientos actuales, sobre nutricion . Washintong, EUA: ILSI.

4 Brown, J. E. (2014). Nutricion en las diferentes etapas de la vida. Mexico: Mc Graw Hill.

5 Castello, C. (2015). Nutricion . Scielo, 1-25 Kennedy ET, Ohls J, Carlso S, Fleming K.The healthy eating index: Desing and Applications Int Am Diet Assoc 2015; 95: 1103-1118.

6. Sánchez Villegas A, Martínez JA, De Irala I, MartínezGonzález MA. Determinants of the adherence to an "a priori"defined Mediterranean dietary pattern Eur J Nutr 2012; 41 (6): 249-257. 7. Tur J, Romaguera D, Pons A. The Diet Quality Index-International (DQI-I): is it a useful tool to evaluate the quality of the Mediterranean diet? Br J Nutr 2015; 93: 369-376

8. Riba i Sicart, M. Estudio de hábitos alimentarios en población universitaria y sus condiciones. 2012. Universidad Autónoma de Barcelona. Facultad de Veterinaria. Dpto Ciencia animal y de los Alimentos, 289 pp. 7.

9. Ortega, R. Nutrición en la población femenina. Desde la infancia a la edad avanzada. Ergón, 2007, 158 pp. 8. Bollat Montenegro, P. y Durá Travé, T. Modelo dietético de los universitarios. Nutric. Hosp. 2010; 23 (6): 619-629.

10. Serrano-Ríos M y Mateos J.A. Nutrición y Alimentación. Nuevas perspectivas. México: McGraw Hill- Interamericana; 2012.

11. Daly JM. Human energy requirements: overestimation by widely used prediction equation. Am J Clin Nutr 2015; 421: 1170-1176

12. McGinnis JM, Foege WH: Actual causes of death in the United Status. JAMA 2013; 270

13. Pi-Sunyer FX: Short-term medical benefits and adverse effects of weight loss. Ann Intern Med 2017 Oct 1; 119(7 Pt 2): 722-6. Review.

14. US Department of Health and Human Services (USDHHS) (2018): 2018 poverty guidelines. Federal Register 63, 9235- 9238. 